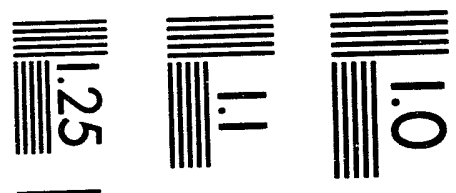

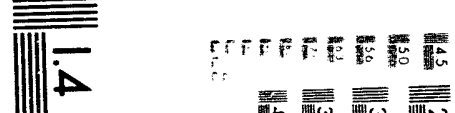

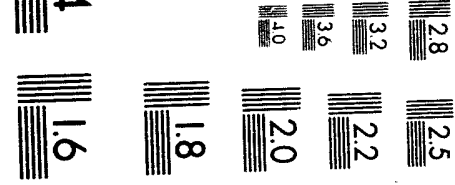



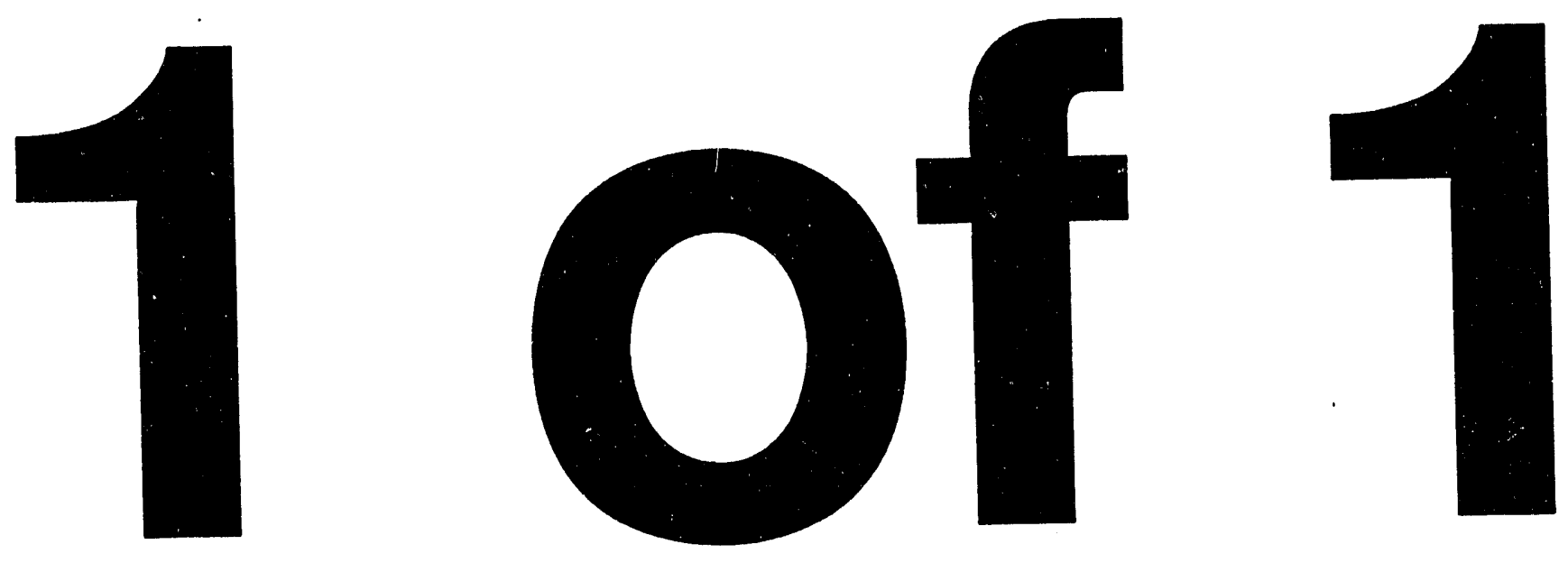
LA-UR - 93-4161

Author(s): $\quad$ Dipen N. Sinha and Stanley T. Kosiewicz

Submitted to: $\quad$ Materials Society Meeting Proceedings

Boston, MA

November 29-December 3, 1993

\section{DISCLAIMER}

This report was prepared as an account of work sponsored by an agency of the United States Government. Neither the United States Government nor any agency thereof, nor any of their employees, makes any warranty, express or implied, or assumes any legal liability or responsibility for the accuracy, completeness, or usefulness of any information, apparatus, product, or bility for the accuracy, completeness, or use would not infringe privately owned rights. Reference herein to any specific commercial product, process, or service by trade name, trademark, manufacturer, or otherwise does not necessarily constitute or imply its endorsement, recommendation, or favoring by the United States Government or any agency thereof. The views and opinions of authors expressed herein do not necessarily state or reflect those of the United States Government or any agency thereof.

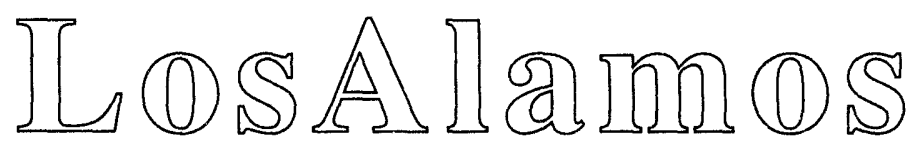

FORM NO. 836 R4 ST, N O $26295 / 81$

\section{Los Alamos National Laboratory Los Alamos,New Mexico 87545 MASTER}




\title{
NON-INVASIVE DETERMINATION OF WASTE DRUM PRESSURIZATION BY ACOUSTIC RESONANCE SPECTROSCOPY
}

\author{
DIPEN N. SINHA* AND STANLEY T. KOSIEWICZ** \\ *Electronic Materials \& Device Research Group, MS D429 \\ **Environmental Restoration Group, MS K570 \\ Los Alamos National Laboratory, Los Alamos, New Mexico 87545
}

\begin{abstract}
Preliminary work suggests that Acoustic Resonance Spectroscopy (ARS) can noninvasively determine gas pressurization of waste drums. This is done by exciting the various mechanical vibrational modes of the test drum and analyzing the characteristics of the resulting resonance frequencies and their higher harmonics. If the contained waste is a liquid, the liquid level and also some properties of the liquid including density can be determined. Additional work is needed to exploit this novel acoustic technique for noninvasive characterization of waste storage drums.
\end{abstract}

\section{INTRODUCTION}

Drums of hazardous, radioactive, and mixed wastes can become pressurized through several different mechanisms [1,2]. Pressurization can pose a safety hazard through potential for fire, explosion or high pressure. Visual inspection of one thousand 55-gallon drums [3] at the Rocky Flats Plant revealed some had bulging lids which could indicate pressurization (presumably from the radiolytic degradation of organic waste matrices.) This finding prompted the US Department of Energy (DOE) Defense Programs (DP) to draft a Safety Accion Letter [4] requiring DP sites to inspect drums for potential pressurization. At present, quaniitative methods for non-invasively determining pressurization are needed but unavilabic

An alternate method to visual inspection for assessing potential $\mathrm{dn}_{1}, \cdots \mathrm{pr}$, ssurization

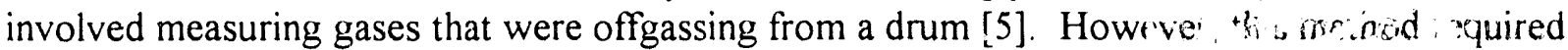
several days of sample collection, whereas, the measurements using the sis tec nisule described here can be carried out in approximately 20 seconds.

\section{MEASUREMENT PRINCIPLES}

We have used the name Acoustic Resonance Spectroscopy (ARS) a a re rieric term to describe a class of acoustic noninvasive testing methods that relies on the mecianical resonant vibrational characteristics of a drum to extract physical information regarding the drum and its contents[6]. The underlying principles of this technique have been known for decades. We have adapted the technique specifically for determining physical properties (e.g., sound speed, density, viscosity etc., ) of fluids inside sealed containers[7]. The development of this technique 
solved the urgent need to verify the contents of chemical munitions for international treaty verifications.

All solid (elastic) objects possess a multitude of natural resonance frequencies. These resonance frequencies depend on the physical nature, such as geometry and elastic properties, of the object. Anything that affects these physical parameters, including external or internal stress, affects the resonance characteristics. Depending on the material and its geometry, mechanical stress can magnify the nonlinearity in the system, and this can easily be detected. Because of the - large number of resonance frequencies involved in any object, it is convenient to refer to this as an acoustic spectrum of a given object. Fluids inside containers can have a pronounced effect on the acoustic spectrum[6]. If the container shell thickness, as in the case of a hollow cylinder, is relatively thin, then the presence of liquid inside tends to shift the resonance frequencies to lower values and also increase the sharpness $(\mathrm{Q})$ of the resonance curves. These effects are not uniform over the entire spectrum but depend on the particular resonance modes. These particular characteristics help to extract meaningful physical parameters of the liquid for containers of simple geometry, such as cylinders.

For 55-gallon drums, where the shell thickness is a small fraction of the radius, one can take advantage of another effect. In metal plates, one mode of sound propagation is by bending (flexural) waves (very similar to ripple waves on water) [8]. The speed of the bending wave $C_{b}$ depends on the thickness $(h)$ of the plate and the frequency $(f)$ as

$$
\mathrm{C}_{\mathrm{b}}=\left(1.8 \cdot \mathrm{C}_{\mathrm{L}} \cdot \mathrm{h} \cdot \mathrm{f}\right)^{1 / 2}
$$

where $C_{L}$ is the speed of the longitudinal wave in the metal. If this metal plate is immersed in a liquid of density $(\rho)$, the propagation of the bending wave is slowed. Ageeva [9] experimentally verified this slowing down effect using an aluminum strip. The relationship between the angular frequency $(\omega$, where $\omega=2 \pi f)$ and the wavenumber $(k$, where $k=2 \pi / \lambda$ and $\lambda$ is the wavelength) of the bending wave in a metal plate in contact with a liquid on one side was shown [8] to be

$$
\omega^{2}=\frac{D k^{5}}{\rho+h \rho_{0} k} ; D=C_{L}\left(\frac{\rho_{0} h^{3}}{12}\right)
$$

where $D$ is the flexural rigidity of the plate and $\rho_{O}$ is the density of the metal plate. The numerator in Eq. 2 shows that if $h$ and $k$ are relatively small, then the effect of the liquid density can be quite measurable. Since a drum can be considered as a rolled plate in the shape of a cylinder, Eq. 2 would apply to the liquid inside the drum. We have taken advantage of this fact to determine liquid fill level and density inside a 55-gallon drum. In the following Sections, we discuss our measurement techniques. All measurements discussed below refer to measurements carried out on a single new 55-gallon drum half filled with water.

\section{NON-INVASIVE DRUM PRESSURIZATION DETERMINATION}

The setup used is shown in Fig. 1. A brand new 55-gallon drum was used. It was approximately half filled with water as the test liquid to determine both fluid level and information about fluid density. The drum lid had a gasket and allowed pressurization of the drum through a tube attached to one of the ports on the lid. The pressurization was done using compressed air. A valve on the attachment allowed depressurization of the system. A 


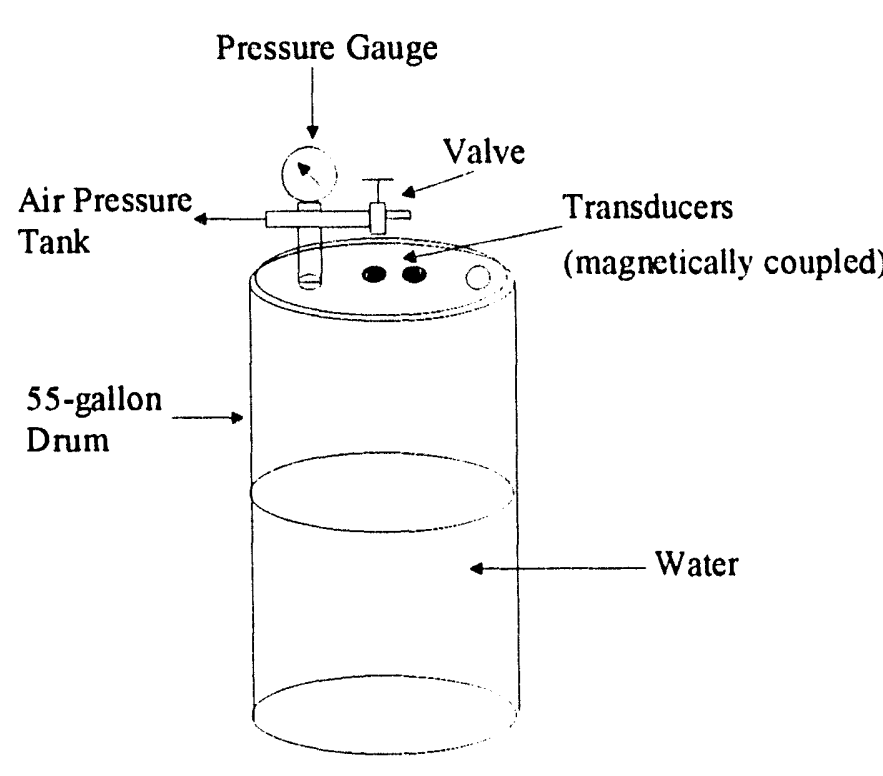

Figure 1 Setup for drum pressurization mechanical dial gauge was used to determine the pressure. Because of leaks, the maximum pressure that could be maintained for a long time with all the valves closed was 300 torr.

The acoustic resonance measurement consists of excitation of the drum (lid or side) using a piezoelectric transducer magnetically coupled to the surface and detection of the drum vibrational response using a second similar transducer. The two transducers are kept approximately 1 inch apart. The electronics consist of a PC plug-in board (NEEL Electronics DSA100 Digital Synthesizer and Analyzer, CA) and a portable computer. A completely field-portable and batteryoperated system is also available. This DSA100 board is completely computer-controlled and provides a swept-sine frequency excitation output. The DSA100 board has the capability to track the second harmonic component of any signal. The frequency range is selectable from 20 $\mathrm{Hz}$ to $100 \mathrm{kHz}$. The maximum drive amplitude is $10 \mathrm{~V}$ peak-to-peak. The signal received from the second transducer is analyzed by the same board and provides a frequency spectrum in a few seconds that is displayed in real-time $\mathrm{cn}$ the computer graphics screen. The electronics in the board simulate a very narrow tracking filter and thus the measurements obtained are almost entirely immune to ambient noise. As the frequency input to the transducer is swept, the metal drum surface it contacts vibrates at that frequency and the magnitude of this vibration is detected by the second transducer. Because of the specific geometry of the drum and the lid, there are a multitude of frequencies where the whole drum structure resonates. These show up as resonance peaks in the acquired frequency spectrum, and are a rich source of information on the drum metal structure, its mechanical properties, and the properties of the fluid inside it. Total measurement time is less than 10 seconds.

If the drum is pressurized, the lid and the whole drum are mechanically stressed. This stress is reflected as shifts in frequency in the resonance spectrum. By comparing these frequency shifts, it is possible to determine drum pressurization. However, this method is difficult to implement because one needs to compare the measurement against the frequency spectrum of the same drum when it is not pressurized. This reference information is not likely to be available. The frequency spectrum of a given drum is, to a large extent, unique to that particular drum and one cannot rely on obtaining a standard spectrum for all similar drums.

We have experimentally found that the elastic behavior of metals become nonlinear when they are stressed. The actual degree of nonlinearity is quite small and it depends on the particular metal and the geometry involved. By nonlinearity we mean that the stress and strain are not linearly dependent on each other. When such a system is excited into mechanical resonance, the vibration amplitude of the response becomes very large and the nonlinearity effects may become detectable. We find this to be the case. 


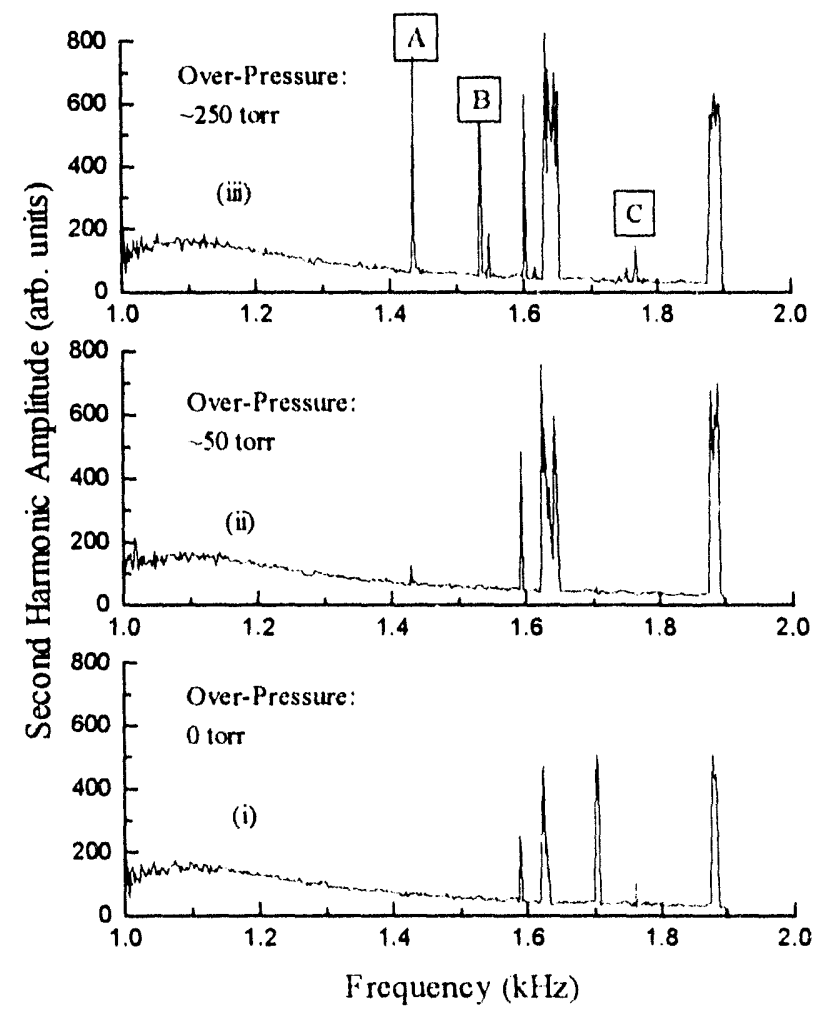

Figure 2. Effect of drun over-pressure on second harmonic amplitude.

Figure 2 shows spectra obtained as a function of drum pressurization. This figure shows the magnitude of the second harmonic as a function of frequency. Fig 2(i) shows the data when there is no over pressure inside the drum. The peaks seen above 1.6 $\mathrm{kHz}$ are probably the result of clamping of the lid which imparts a lot of stress on the lid edge. Figure 2(ii) shows the effect of slight ( $\sim 50$ torr) over pressure. Note the appearance of a small peak slightly above $1.4 \mathrm{kHz}$ and some qualitative changes in the peaks above $1.6 \mathrm{kHz}$. As the pressure is slowly raised in small steps and held (not all over-pressure data are shown), the amplitude of the second harmonic peak grows monotonically. At an overpressure of approximately 250 torr, the peak marked as (A) has grown very significantly. Some secondary peaks, marked $\mathrm{B}$ and $\mathrm{C}$, also appear. This appearance of second harmonic peaks at low frequency with over-pressure is

completely reproducible.

We find that the appearance of asecond harmonic at low pressures below $1.5 \mathrm{kHz}$ is a distinctive signature of drum over-pressurization. The advantage of our method is that we are not comparing subtle shifts in resonance frequencies to determine over-pressure, but are simply examining the resonance spectra for the appearance of second harmonic peaks at low frequency. At low frequency one observes only a very small number of resonance peaks closer to the fundamental modes of vibration of the lid and this can be easily monitored. Although, this effect is also present at high frequencies, it is difficult to isolate because of the large number of closely spaced peaks.

\section{LIQUID LEVEL AND LIQUID FILL PROPERTY DETERMINATION:}

Figure 3 shows the resonance spectrum of the drum with the transducers on the side wall. For clarity, only a small frequency range is shown. Measurements were made with both transducers (parallel to the liquid level and 1 inch apart) below and above the water level. The noisy looking data are the result of numerous resonance modes of the drum. The presence of the water loads the drum to cause large, easily detectable frequency shifts $(\sim 5000 \mathrm{~Hz})$ of the appropriate modes. This is particularly apparent between 15 and $30 \mathrm{kHz}$ (note the separation between the two datasets). There is a large shift in the overall spectrum at that location.

This spectrum also shows that the periodic nature of the resonances (circumferential modes) is clearly affected by the liquid. As discussed previously, the propagation speed of the bending waves is affected by the liquid in contact with the surface. This produces the difference 


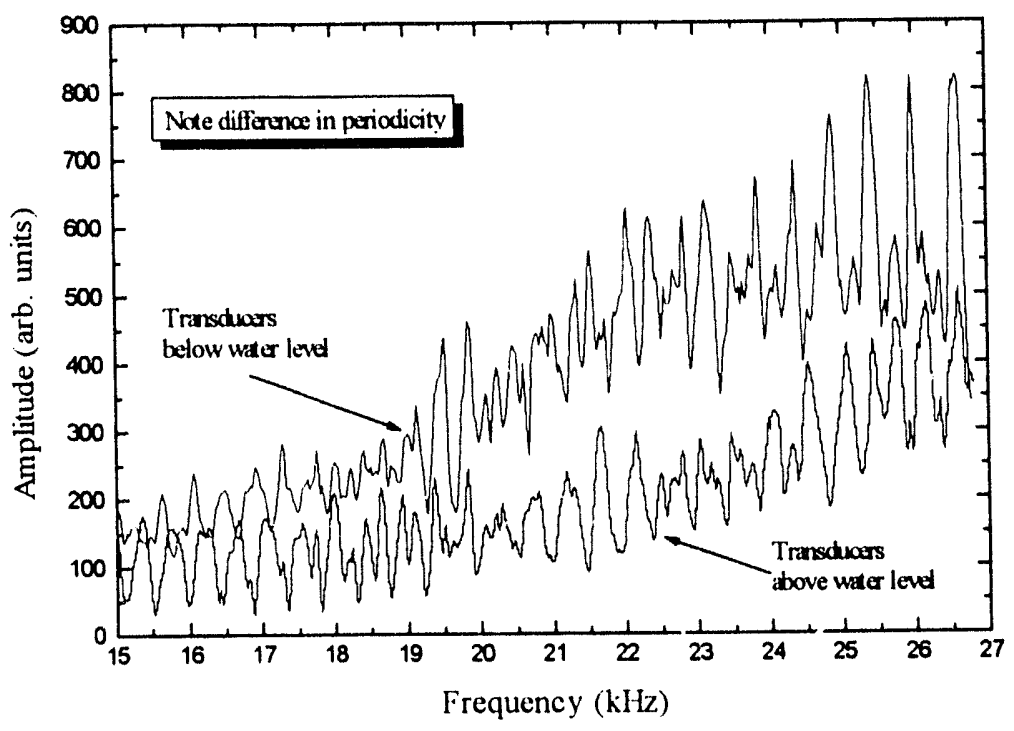

Figure 3. Acoustic spectra of a 55-gallon drum. in periodicity shown in

Fig. 3. A quick way to observe this difference is to examine the autocorrelation of each data-set. A peak in the autocorrelation represents the periodicity in terms of frequency. The presence of water produces a shift of approximately $150 \mathrm{~Hz}$ This is quite large and easily detectable. We have independently and directly verified the slowing down of the bending waves below

the water level by using a tone-burst technique. In this technique, approximately 10 cycles of a sine-wave of any desired frequency is applied to one transducer which excites the bending waves on the drum surface. The arrival of this wave is detected by the second transducer. We found the delay in arrival was indeed longer for measurements made below the water level as compared to that in air. We repeated the measurements over a large frequency range ( $1-100$ $\mathrm{kHz}$ ) and found the results to be consistent with Eqs. 1 and 2. Eq. 2 shows that the wave propagation speed is related to the liquid density. Consequently, this method can provide a determination of the density of the liquid inside the drum. It can also resolve variations in density, such as stratification, as a function of height such as stratification. If there is some knowledge of the contents, it may be possible to use this technique to sort items according to liquid property.

\section{CONCLUSIONS AND RECOMMENDATIONS}

The information presented in this paper should be considered as preliminary, qualitative and only as proof of principle of the various concepts discussed. While further research is warranted to refine this technique, the underlying theory is well understood. The measurements need to be validated by studies on a larger number of drums, both old and new. Clearly, acoustic resonance techniques can be used to obtain a distinctive signature related to over pressurization of a waste drum. It may be possible to measure the bending wave speed under stress due to over-pressurization to see if they are different. If the over-pressurization affects the bending wave propagation speed, then that can provide an additional complementary method for over-pressurization detection.

The slowing down of the bending wave propagation in the metal shell of the drum due to the presence of the liquid can be a powerful tool in studying liquid contents of waste storage drums. Measurements should be repeated with various liquids of different densities or using the same liquid but varying its density by mixing other materials in it. Simple, handheld, field- 
portable instrumentation can be built for monitoring waste storage drums based on the methods described in this paper. The main advantage of the techniques presented is that, these do not need prior calibration when measurement such as fill level is required.

For determining liquid fill level and liquid density, the direct tone-burst approach is more suitable than the resonance method because the measurement is unambiguous. In the case of the resonance approach, it is difficult to isolate the circumferential (horizontal) modes from the axial (vertical) modes. These modes are superimposed on each other. In the axial modes, two propagation speeds (below and above water level) are mixed up. An appropriate algorithm can separate this information out and such algorithms need to be developed.

Finally, more rigorous mathematical modeling needs to be carried out to explain all the effects observed in a more quantitative manner. We feel that the methods described are simple to implement and can provide the necessary tool for storage drum monitoring.

This work was supported in part by the Defense Nuclear Agency.

\section{REFERENCES}

1. Fire Explosion, and High-Pressure Hazards Associated with Waste Drums and Containers, U.S. DOE, Office of Nuclear Safety, Safety Notice DOE/NS-0013, No, 93-1.

2. Waste Management 1992 Symposium Session, Gas Issues related to Nuclear Waste Storage, Tucson, AZ, 1992.

3. L. G. Peppers and T. R. Hergert, Inspection of Drums in Building 371, EG\&G Rocky Flats Plant Internal memorandum, October 15, 1992.

4. Pressurization of Drums, U.S. DOE, Defense Programs, Safety Action letter (Draft) No. 9301, May 1993.

5. S. T. Kosiewicz and M. Pannel, presented at the 205th ACS National Meeting, Denver, CO, 1993 (unpublished).

6. D. N. Sinha, IEEE Potentials, 11,10 (1992).

7. D. N. Sinha, presented at the 113th ASME Winter Annual Meeting, Anaheim, CA, 1992, paper No. NLTT-4.

8. L. D. Landau and E. M. Lifshitz. Theory of Elasticity, 3rd ed. (Pergamon Press, New York, 1986), p. 99.

9. N. S. Ageeva, Sov. Phys.-Acoustics, 6(1),116 (1960). 

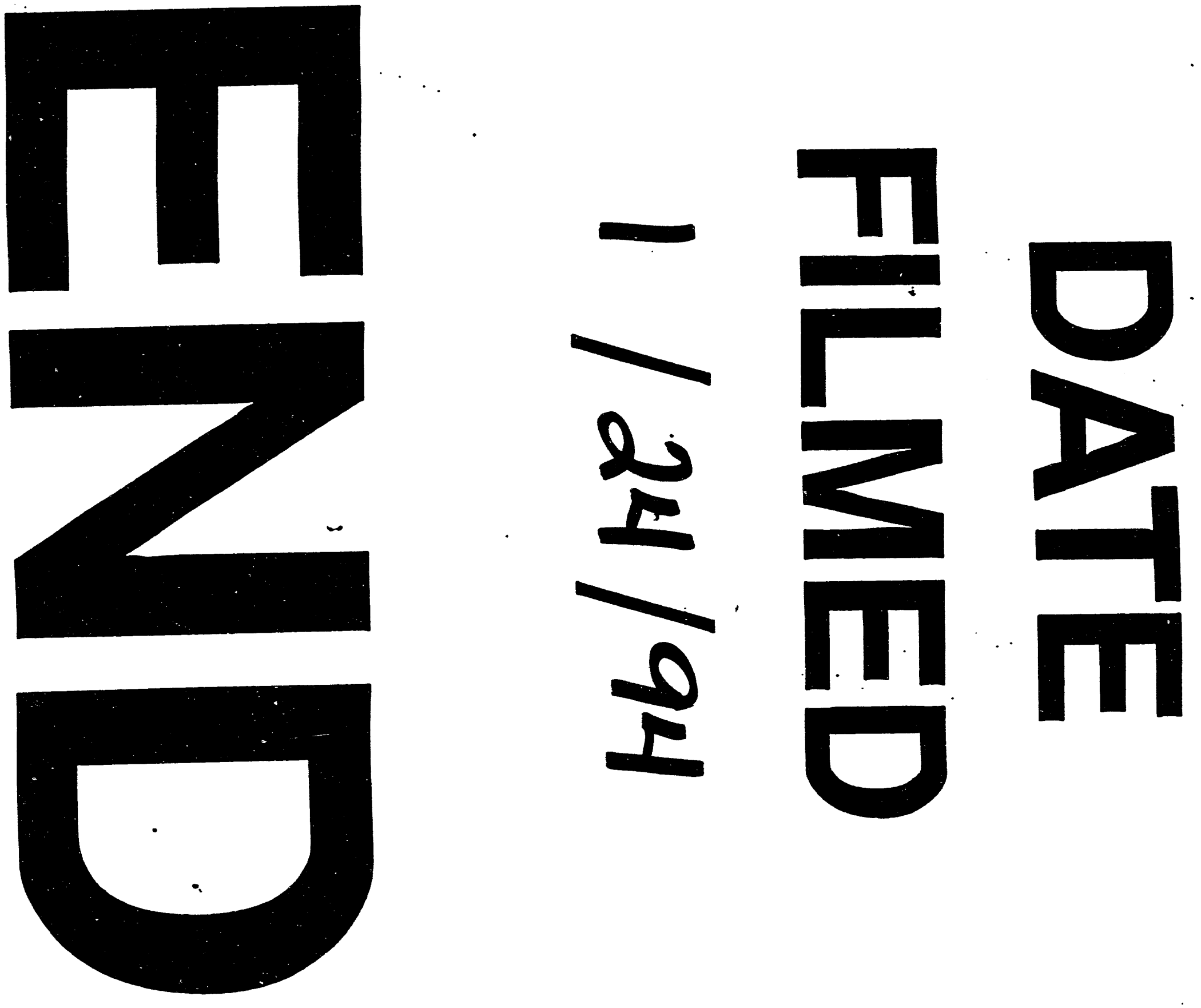
$=$ 